\title{
MEDICINAL PLANTS OF THE GENUS SAPINDUS (SAPINDACEAE) - A REVIEW OF THEIR BOTANY, PHYTOCHEMISTRY, BIOLOGICAL ACTIVITY AND TRADITIONAL USES
}

\author{
Goyal Sachin ${ }^{1 *}$, Kumar Dileep ${ }^{1}$, Menaria Gopal ${ }^{1}$, Singla Shivali ${ }^{1,2}$ \\ ${ }^{1}$ Department of Pharmacology, Pacific College of Pharmacy, Udaipur, Rajasthan (India) \\ 2 Department of Pharmacology, SMS Medical College, Jaipur, Rajasthan (India)
}

Objective: Sapindus is a genus of Sapindaceae family comprises three major species: The American spices, Sapindus saponaria and two Asian species, Sapindus mukorossi and Sapindus trifoliatus and are well known for their folk medicinal values. Several important studies have been published in the intervening years, which have prompted us to reassess Sapindus. In the current review, we provide a comprehensive over view on the botany, traditional uses, phytochemistry and biological activities of the species of Sapindus.

Methods: A literature survey was performed by searching the scientific data bases Pubmed, Google Scholar, SciFinder, Scopus and Web of Science, in addition to traditional Indian medicine and Ayurvedic texts for information on Sapindus.

Results: Plants of the genus Sapindus have been used in traditional medicine for the treatment of ulcers, external wounds, inflammation, epilepsy, dental caries, arthritis, joint pain, gout and rheumatism. Phytochemical studies of this genus have identified more than 103 compounds. Of these compounds, triterpenoidal saponins of oleanane, dammarane and tirucullane are regarded as the active group that is most likely to be responsible for the observed biological activities. The crude extracts, as well as the isolated compounds, from the genus possess antimicrobial, anticancer, spermicidal, hepatoprotective, antioxidant, anti-inflammatory, anti-platelet aggregation, anti-hyperlipidemic, anti-migraine, anti-diabetic, anti-ulcerogenic and analgesic properties.

Conclusion: It is evident from the available literature that Sapindus species possess potential for use as a beneficial therapeutic remedy. Nevertheless, there is clearly a need for further studies focusing on in-vivo with emphasis on molecular mechanisms and eventually clinical trials.
\end{abstract}

Keywords: Biological activities, Botany, Phytochemistry, Sapindus, Saponins

\section{INTRODUCTION}

Sapindus is a genus of shrubs and small trees in the Sapindaceae family ${ }^{1,2}$. The genus comprises three major species: The American spices, Sapindus saponaria and two Asian species, Sapindus mukorossi and Sapindus trifoliatus. The American species Sapindus saponaria is popularly known as western soapberry, cherrioni, jaboncillo, sabao-de-macaco, saboeiro, saboneteiro, fruta de sabao, sabao-de-soldado and grows on clay soils and on dry limestone uplands from southwestern Missouri to Louisiana, and westward through Oklahoma and Texas to southern Colorado, New Mexico, southern Arizona, northern Mexico and Brazilian Amazon region ${ }^{3}$. Asian species, Sapindus mukorossi and Sapindus trifoliatus are known as soapnut, soapberry, washnut, reetha, aritha, dodan, doadni and flourishes well in deep clay loamy soil with an annual rainfall of 150-200 mm. Sapindus mukorossi is widely distributed in upper reaches of IndoGangetic plains, Shivaliks and sub Himalayan tracts at altitudes from $200 \mathrm{~m}$ to $1500 \mathrm{~m}$, while Sapindus trifoliatus is commonly found in the Western Ghats and plains of South India ${ }^{4,5}$.

The members of genus Sapindus are well known for their folk medicinal values. The fruit of $S$. saponaria is used (C) 2011-14, JDDT. All Rights Reserved by loacal population for curing ulcers, external wounds and inflammation ${ }^{6}$. S. mukorossi is well known for its detergent and insecticidal properties and it is traditionally used for removing lice from the scalp. The fruits are of considerable importance for their medicinal value for treating a number of diseases like excessive salivation, pimples, epilepsy, chlorosis, migranes, eczema and psoriasis. In Japan its pericarp is called "enmei-hi", which means "life prolonging pericarp" and in China "wu-huan-zi", the "non-illness fruit". The powdered seeds are employed in the treatment of dental caries, arthritis, common colds, constipation and nausea. The leaves are used in baths to relieve joint pain and the roots are used in the treatment of gout and rheumatism ${ }^{7-11}$.

* Corresponding author: Dr. Sachin Goyal

Associate Professor (Pharmacology) Pacific College of Pharmacy, Pacific University Udaipur-313003, Rajasthan (India) Contact: +91-7568918770, +91-294-2494508 Fax: +91-294-2494509, Email: goyalsachin14@gmail.com 
Fruits of $S$. trifoliatus have been considered as a tonic, stomachic, alexipharmic, astringent and sedative to the uterus and also useful in chronic dysentery, diarrhea, cholera, hamicrania, paralysis and epileptic fits of children. The roots used as a collyrium in sore eyes and opthalmia. The seeds are employed to stimulate the uterus in childbirth and to increase mensuration ${ }^{5,7,12-14}$.

Recently, a number of studies have been conducted on the phytochemical and biological activities aspects of Sapindus. Phytochemical studies of Sapindus plants have identified more than 103 compounds, including flavonoids, triterpenoids, glycosides, carbohydrates, fatty acids, phenols, fixed oil, and saponins. Of these compounds, saponins are regarded as the active group that is most likely to be responsible for the observed biological effects ${ }^{4,7,15-17}$. Moreover, both in-vivo and invitro experiments have demonstrated that Sapindus exhibits a diverse set of antimicrobial, anticancer, spermicidal, hepatoprotective, anxiolytic, antioxidant, Anti-inflammatory, anti-platelet aggregation, antifertility, anti-hyperlipidemic, anti-migraine, anti-diabetic, anti-pruritis, anti-ulcerogenic and analgesic biological effects $4,7,12,16-18$. These studies will be evaluated in detail in this review.

Several important studies have been published in the intervening years, which have prompted us to reassess Sapindus. In the current review, we provide a comprehensive over view on the ethnopharmacology, phytochemistry and biological activities of the species of Sapindus.

\section{BOTANICAL DESCRIPTIONS}

The genus Sapindus is classified in the division Magnoliophyta, class Magnoliopsida, subclass Rosidae, order Sapindales and family Sapindaceae ${ }^{13,16,17}$.

The three species of the genus are the American Sapindus saponaria and two Asian species, Sapindus mukorossi and Sapindus trifoliatus. Sapindus species are deciduous trees that thrive in tropical climates. Sapindus saponaria is a well known plant in American region, and has been used as traditional medicine by local population since ancient times. Sapindus saponaria is widely distributed with populations reported in southwestern Missouri to Louisiana, and westward through Oklahoma and Texas to southern Colorado, New Mexico, southern Arizona, northern Mexico and Brazilian Amazon region 3,6 .

Sapindus saponaria is a small to medium deciduous tree, 7.7 to $15.4 \mathrm{~m}$ tall. The heavy, strong, close grained wood splits into thin strips. It contains long, pinnate leaves. This plant is described as polygamo-dioecious. That is, individual trees in a population may be truly dioecious (having only male or female flowers) or they may contain flowers with both male and female functions. The small, white flowers, borne in rather large clusters of terminal or axillary panicles, open during May to July. The fruit, a yellow, translucent, globular drupe measuring 10 to $14 \mathrm{~mm}$ in diameter, usually contains a single, dark brown, hard-coated seed, but occasionally 2 or 3 seeds are present. The fruits ripen during September to October and persist on the tree until late winter or spring ${ }^{19,20}$.
Sapindus mukorossi is a tree of tropical and sub-tropical region of Asia. It is a deciduous tree widely grown in upper reaches of Indo-Gangetic plains, Shivaliks and sub Himalayan tracts at altitudes from $200 \mathrm{~m}$ to $1500 \mathrm{~m}$. The Sapindus mukorossi is a fairly large tree with a straight trunk up to 12 meters in height, sometimes attaining a height of $20 \mathrm{~m}$ and a girth of $1.8 \mathrm{~m}$, with a globose crown and rather fine leathery foliage. Bark is dark to pale yellow, fairly smooth, with many vertical lines of lenticels and fine fissures exfoliating in irregular wood scales. The blaze is $0.8-1.3 \mathrm{~cm}$, hard, not fibrous, pale orange brown, brittle and granular. Leaves are $30-50 \mathrm{~cm}$ long, alternate, paripinnate; common petiole very narrowly bordered, glabrous; leaflets 5-10 pairs, opposite or alternate, $5-18$ by $2.5-5 \mathrm{~cm}$, lanceolate, acuminate, entire, glabrous, often slightly falcate or oblique; petioles 2-5 $\mathrm{m}$ long. Inflorescence is a compound terminal panicle, $30 \mathrm{~cm}$ or more in length, with pubescent branches. Flowers are about $5 \mathrm{~mm}$ across, small, terminal, polygamous, greenish white, subsessile, numerous, mostly bisexual. Sepals 5 , each with a woolly scale on either side above the claw. Fruits are globose, fleshy, 1-seeded drupe, sometimes two drupels together, about 1.8-2.5 cm across. Seeds are $0.8-1.3 \mathrm{~cm}$ in diameter, globose, smooth, black and loosely placed in dry fruit ${ }^{4,16}$.

The third species, Sapindus trifoliatus, occurs in the Western Ghats and plains of South India. This is a deciduous tree and flourishes well in deep clay loamy soil with an annual rain fall of $200 \mathrm{~mm}$. It can reach a height of $25 \mathrm{~m}$. Leaves are alternate, $15-40 \mathrm{~cm}$ long, pinnate, with 14 - 30 leaflets and the terminal leaflet often absent. The leaflets are elliptic-lance shaped, smooth with tipped points and slightly oblique based. They are often found in pairs of 2 or 3 and are 8 to $18 \mathrm{~cm}$ long and 5 to $7.5 \mathrm{~cm}$ wide. The flowers form in large panicles. The flowers are small greenish white in color and open during November to January. The fruit is a small leathery-skinned drupe $1-2 \mathrm{~cm}$ in diameter. The fruits are solitary globose appears in the month of JulyAugust. The fruit is velvety when young and turns hard and smooth on maturing. The fruits and seeds are slightly smaller than the north Indian species $5,13,21$.

\section{TRADITIONAL USES}

Sapindus species have a long history as traditional remedies (Table 1) ${ }^{5-14}$. All these species have been used in the areas where they are naturally distributed. Sapindus saponaria is mainly used in American region, while Sapindus mukorossi and Sapindus trifoliatus are mainly used in Asian countries as a traditional remedy. Interestingly, all these species have been used as detergent.

\section{Sapindus saponaria}

The fruit of S. saponaria, is used by local population for curing ulcers, external wounds and inflammations ${ }^{6}$.

\section{Sapindus mukorossi}

Sapindus mukorossi is well known for its folk medicinal values. Pericarps of Sapindus mukorossi have been traditionally used as an expectorant as well as a source of natural surfactant ${ }^{9}$. Due to the presence of saponins, 
soapnut is well known for its detergent and insecticidal properties and it is traditionally used for removing lice from the scalp. The fruits are of considerable importance for their medicinal value for treating a number of diseases like excessive salivation, pimples, epilepsy, chlorosis, migranes, eczema and psoriasis. In Japan its pericarp is called "enmei-hi", which means "life prolonging pericarp" and in China "wu-huan-zi", the "non-illness fruit". The powdered seeds are employed in the treatment of dental caries, arthritis, common colds, constipation and nausea. The seeds of are used in Ayurvedic medicine to remove tan and freckles from the skin. It cleanses the skin of oily secretion and is even used as a cleanser for washing hair as it forms a rich, natural lather. The leaves are used in baths to relieve joint pain and the roots are used in the treatment of gout and rheumatism ${ }^{7-11}$. Since ancient times, Sapindus mukorossi has been used as a detergent for shawls and silks. The fruit was utilized by Indian jewelers for restoring the brightness of tarnished ornaments made of gold, silver and other precious metals ${ }^{10}$.

\section{Sapindus trifoliatus}

The plant is very commonly used in Indian Ayurvedic healing system. It is also used in Unani and Tibetan indigenous medicine ${ }^{13}$. In folklore practice, some of the tribes of Orissa (India) use the aerial parts of this plant for the treatment of diabetes mellitus. Fruits of Sapindus trifoliatus have been considered as a tonic, stomachic, alexipharmic, astringent and sedative to the uterus and also useful in chronic dysentery, diarrhea, cholera, hamicrania, paralysis and epileptic fits of children. The roots used as a collyrium in sore eyes and opthalmia. The seeds are employed to stimulate the uterus in childbirth and to increase mensuration ${ }^{5,7,12-14}$.

\section{Table 1: Species of Sapindus and its traditional uses}

\begin{tabular}{|l|l|l|l|}
\hline Species & Common names & Traditional uses & Ref \\
\hline $\begin{array}{l}\text { Sapindus saponaria L } \\
\text { (Hook. \& Arn.) } \\
\text { Benson }\end{array}$ & $\begin{array}{l}\text { Western soapberry, Cherrioni, } \\
\text { Jaboncillo, Sabao-de-macaco, } \\
\text { Saboeiro, Saboneteiro }\end{array}$ & $\begin{array}{l}\text { Curing ulcer } \\
\text { External wounds } \\
\text { Inflammation }\end{array}$ & 3,6 \\
\hline $\begin{array}{l}\text { Sapindus mukorossi } \\
\text { L.Gaertn. }\end{array}$ & $\begin{array}{l}\text { Soapnut, Soapberry, Washnut, } \\
\text { Reetha, Aritha, Dodan, Doadni }\end{array}$ & $\begin{array}{l}\text { As expectorant, Source of natural surfactant } \\
\text { Insecticidal, treating excessive- salivation, } \\
\text { pimples, epilepsy, chlorosis, migrane, eczema, } \\
\text { psoriasis, dental caries, arthritis common cold, } \\
\text { constipation, nausea, joint pain, gout and } \\
\text { rheumatism }\end{array}$ \\
& & $\begin{array}{l}\text { considered tonic, stomachic, alexipharmic, } \\
\text { astringent and sedative to uterus; treating } \\
\text { diabetes, chronic dysentery, diarrhea, cholera, } \\
\text { paralysis and epileptic- fits of children's; used as } \\
\text { collyrium in sore eyes, opthalmia, to induce } \\
\text { L. trifoliatus }\end{array}$ \\
& $\begin{array}{l}\text { Soapnut, Soapberry, Washnut, } \\
\text { Reetha, Aritha, Dodan, Doadni }\end{array}$ \\
& & $\begin{array}{l}\text { labour pain and normal contraction of uterus after } \\
\text { child birth }\end{array}$ \\
\hline
\end{tabular}

\section{PHYTOCHEMISTRY}

Phytochemical studies of Sapindus plants have identified more than 103 compounds, including flavonoids, triterpenoids, glycosides, carbohydrates, fatty acids, phenols, fixed oil, and saponins. Of these compounds, saponins are regarded as the active group that is most likely to be responsible for the observed pharmacological effects. These saponins are found throughout the plant but are especially concentrated in the fruit 4, 7, 15-17. Saponins are secondary plant metabolites with divergent biological activities ${ }^{23}$. Sapindus saponins are a mixture of six sapindosides (sapindosides A, B, C, D and mukorozi saponins (E1 and Y1), with sapindoside B as one of the major constituents, and identified by liquid chromatography and mass spectroscopy ${ }^{24}$. Saponins are a large family of structurally-related compounds of steroid or triterpenoid aglycone (sapogenin) linked to one or more oligosaccharide moieties by glycosidic linkage. The aglycone, or sapogenin, may contain one or more unsaturated $\mathrm{C}-\mathrm{C}$ bonds. The oligosaccharide chain is normally attached at the $\mathrm{C} 3$ position (monodesmosidic), but many saponins have an additional sugar moiety at the
$\mathrm{C}_{2,6}$ or $\mathrm{C}_{2,8}$ position (bidesmosidic). The great complexity of the saponin structure arises from the variability of the aglycone structure, the nature of the side chains and the position of attachment of these moieties on the aglycone 23. The carbohydrate moiety consists of pentoses, hexoses or uronic acids. Due to this complexity, saponins are difficult to classify. Because it is no longer customary to classify compounds based on their physicochemical or biological properties, a state of the art classification based on the biosynthesis of the saponin carbon skeletons was proposed by Vincken et $\mathrm{al}^{25}$.

Different types of triterpene, saponins of oleanane, dammarane and tirucullane type were isolated from the galls, fruits and roots of Sapindus species. Oleanane type triterpenoid saponins named Sapindoside A \& B (Fig. 34 $\& 35)$ were reported from the fruits ${ }^{26}$. Sapindoside C (Fig. 36) ${ }^{27}$, Sapindoside D (Fig. 37) ${ }^{28}$, which is a hexaoside of hederagenin, and Sapindoside E (Fig. 38) ${ }^{29}$, a nonaoside of hederagenin, was isolated and identified from the methanolic extract of the fruits of Sapindus species. 
Dammarane-type saponins, named Sapinmusaponins A \& B (Fig. 11 \& 12), C-E (Fig. 15, 16, 17), together with three known phenylpropanoid glycosides, were isolated from the galls of Sapindus mukorossi ${ }^{30}$. Tirucallane-type saponins, sapinmusaponins F-J (Fig. 18-22), were isolated from the galls as reported by Huang et $\mathrm{al}^{31}$. The structures of these saponins were elucidated on the basis of spectroscopic analysis including 1D and 2D NMR techniques.

Triterpene saponins of oleanane type like, Sapinmusaponin K-N (Fig. 25-28), Mukorozisaponin G \& E1 (Fig. 29-30), Sapindoside A \& B along with dammarane types like Sapinmusaponin O and P (Fig. 13 $\& 14)$ were isolated from fruits and the galls of Sapindus mukorossi as per Huang et al ${ }^{32}$. In another study by Nakayama et al., Mukorozisaponin Y1 (Fig. 31), Y2 (Fig. 32), X (Fig. 33) were isolated from the pericarp ${ }^{33}$.
Fractionation of an ethanolic extract of the galls of Sapindus mukorossi has resulted in the isolation of two tirucallane type triterpenoid saponins, sapinmusaponin Q and R (Fig. 23-24), along with three known oleanane type triterpenoid saponins: sapindoside A, sapindoside B, and hederagenin-3-O-[ $\beta$-D-xylopyranosyl- $(1 \rightarrow 3)]-\alpha-\mathrm{L}-$ rhamnopyranosyl- $(1 \rightarrow 2)+-\alpha-L$ arabinopyranoside ${ }^{34}$. The roots of Sapindus mukorossi contain tirucallane-type triterpenoid saponins like Sapimukoside A \& B ${ }^{35}$ and Sapimukoside C \& D ${ }^{36}$. Further investigation of the roots of Sapindus mukorossi by $\mathrm{Ni}$ et al., reported the presence of Sapimukosides E-J ${ }^{37}$. The structures of Sapimukosides A-J are shown in Fig. 1-10 respectively. Table 2 shows whole view of all the saponins isolated from genus Sapindus.

Table 2: List of saponins isolated from genus Sapindus

\begin{tabular}{|c|c|c|c|c|}
\hline Saponins & Chemical name & $\begin{array}{l}\text { Oleanane/Tirucullane/ } \\
\text { Dammarane type }\end{array}$ & Structure & Ref \\
\hline $\begin{array}{l}\text { Sapindoside } \\
\text { A }\end{array}$ & $\begin{array}{l}\text { Hederagenin-3-O- } \alpha \text {-L-arabinosyl- } \\
(2 \rightarrow 1) \text { - } \alpha \text {-L-rhamnopyranoside }\end{array}$ & Oleanane & 34 & 26 \\
\hline B & $\begin{array}{l}(2 \rightarrow 1) \text { - } \alpha \text {-L-rhamnopyranoside } \\
\text { Hederagenin-3-O- } \alpha \text {-L-arabinosyl- } \\
(2 \rightarrow 1) \text {-O- } \alpha \text {-L-rhamnopyranosyl- }(3 \rightarrow 1) \text { - } \\
\beta \text {-D-xylanopyranoside }\end{array}$ & Oleanane & 35 & 26 \\
\hline $\mathrm{C}$ & $\begin{array}{l}\text { Hederagenin-3-O- } \beta \text {-D-glucosyl }(1 \rightarrow 4)- \\
\beta \text {-D-xylosyl }(1 \rightarrow 3) \text { - } \alpha \text {-Lrhamnosyl }(1 \rightarrow 2) \text { - } \\
\alpha \text {-L-arabinoside }\end{array}$ & Oleanane & 36 & 27 \\
\hline Sapinmusaponin & & & & \\
\hline A & $\begin{array}{l}\text { 3,7,20(S),22-tetrahydroxydammar-24-ene-3-O-a- } \\
\text { L-rhamnopyranosyl-( }(\rightarrow 2) \text {-D- glucopyranoside }\end{array}$ & Dammarane & 11 & 30 \\
\hline B & $\begin{array}{l}\text { 3,7,20(S),22,23-pentahydroxydammar-24-ene-3- } \\
\text { O-a-L-rhamnopyranosyl-( }(1 \rightarrow 2) \text {-D } \\
\text { glucopyranoside }\end{array}$ & Dammarane & 12 & 30 \\
\hline $\mathrm{C}$ & $\begin{array}{l}\text { 3,7,20(S),22,25-pentahydroxydammar-23- } \\
\text { ene-3-O-a-L-rhamnopyranosyl-(1 }(\rightarrow 2) \text {-D- } \\
\text { glucopyranoside }\end{array}$ & Dammarane & 15 & 30 \\
\hline $\mathrm{D}$ & $\begin{array}{l}\text { 25-methoxy-3,7,20(S),22- } \\
\text { tetrahydroxydammar-23-ene-3-O-a-L- } \\
\text { rhamnopyranosyl- }(1 \rightarrow 2) \text {-D-glucopyranoside }\end{array}$ & Dammarane & 16 & 30 \\
\hline $\mathrm{E}$ & $\begin{array}{l}\text { 25-methoxy-3,7,20(R)-trihydroxydammar- } \\
\text { 23-ene-3-O-a-L-rhamnopyranosyl-( } 1 \rightarrow 2) \text {-D- } \\
\text { glucopyranoside }\end{array}$ & Dammarane & 17 & 30 \\
\hline $\mathrm{F}$ & $\begin{array}{l}21 \beta \text {-methoxy-3- } \beta-21(\mathrm{~S}), 23 \mathrm{I}-\mathrm{epoxy} \\
\text { tirucall-7,24-diene-3-O- } \alpha \text {-L-rhamnopyranosyl- } \\
(1 \rightarrow 6)-\beta-\mathrm{D} \text { - glucopyranosyl }\end{array}$ & Tirucullane & 18 & 31 \\
\hline G & $\begin{array}{l}21 \alpha \text {-methoxy-3- } \beta-21(\mathrm{~S}), 23 \mathrm{I}-\mathrm{epoxy} \\
\text { tirucall-7,24-diene-3-O- } \alpha \text {-L- } \\
\text { rhamnopyranosyl-( }(1 \rightarrow 6)-\beta-\mathrm{D} \text { - glucopyranosyl }\end{array}$ & Tirucullane & 19 & 31 \\
\hline $\mathrm{H}$ & $\begin{array}{l}21 \alpha \text {-methoxy-3- } \beta \text { - } 21(\mathrm{~S}), 23 \mathrm{I}-\mathrm{epoxy} \\
\text { tirucall-7,24-diene-3-O- } \alpha \text {-L- } \\
\text { rhamnopyranosyl-( }(1 \rightarrow 2)-\beta \text {-D- glucopyranosyl }\end{array}$ & Tirucullane & 20 & 31 \\
\hline I & $\begin{array}{l}21 \beta \text {-methoxy-3- } \beta-21(\mathrm{~S}), 23 \mathrm{I} \text {-epoxy } \\
\text { tirucall-7,24-diene-3-O- } \alpha \text {-L- } \\
\text { dirhamnopyranosyl- }(1 \rightarrow 2,6)-\beta \text {-D- glucopyranosyl }\end{array}$ & Tirucullane & 21 & 31 \\
\hline $\mathrm{J}$ & $\begin{array}{l}21 \alpha-\text { methoxy-3- } \beta-21(\mathrm{~S}), 23 \mathrm{I}-\text {-epoxy tirucall-7,24- } \\
\text { diene-3-O- } \alpha \text {-L- dirhamnopyranosyl- }(1 \rightarrow 2,6)-\beta-\mathrm{D}- \\
\text { glucopyranosyl }\end{array}$ & Tirucullane & 22 & 31 \\
\hline $\mathrm{K}$ & hederagenin-3-O-(3-O-acetyl-alpha-L- & Oleanane & 25 & 32 \\
\hline
\end{tabular}




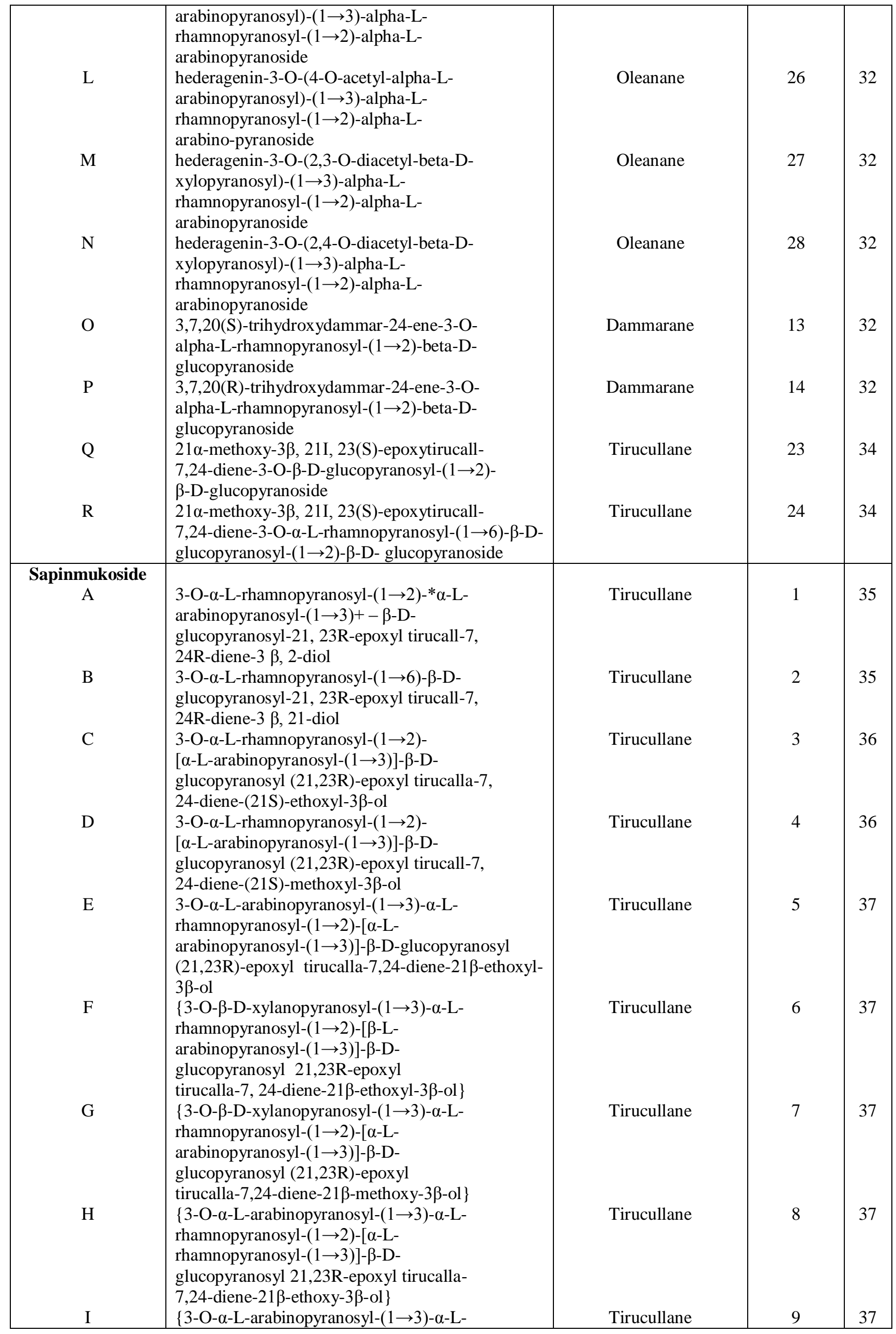




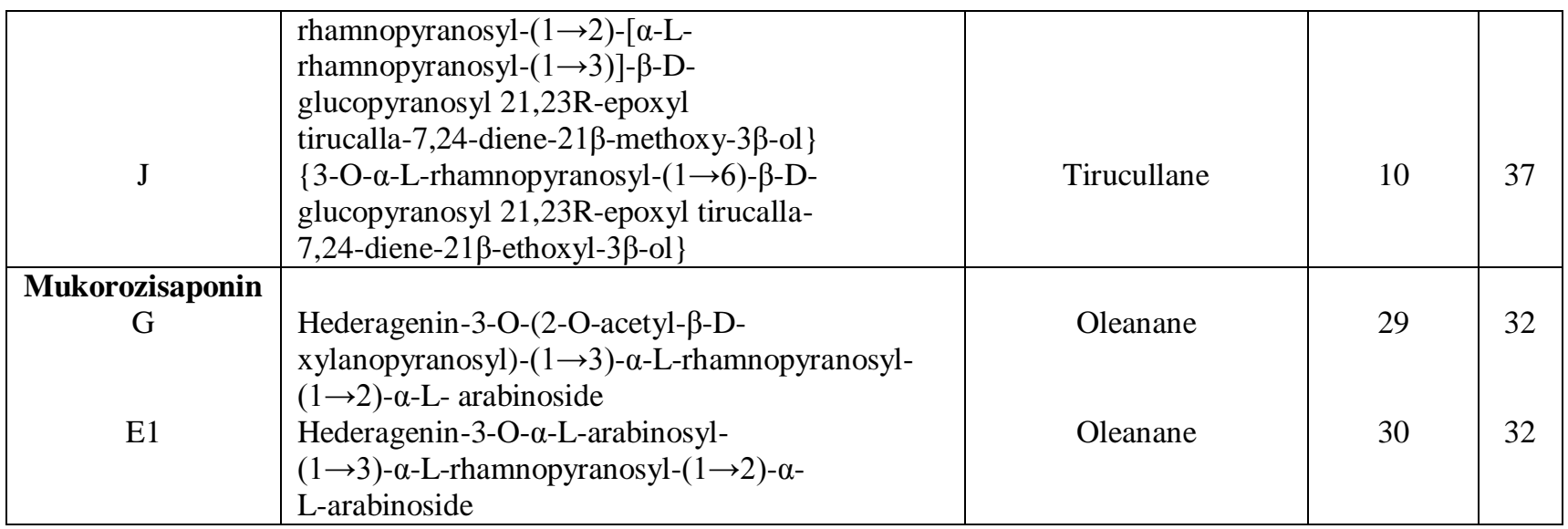

Figure 1: Structure of sapimukosides A-J

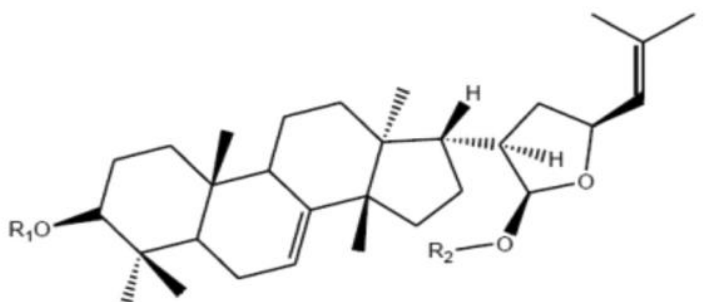

\begin{tabular}{|c|c|c|}
\hline Fig & R1 & $\mathbf{R} 2$ \\
\hline 1 & $\begin{array}{ll}\text { Glc } & \text { 3-Ara } \\
& \text { 2-Rha }\end{array}$ & $\mathbf{H}$ \\
\hline 2 & Glc $_{6}$-Rha & $\mathbf{H}$ \\
\hline 3 & $\begin{array}{ll} & \text { 3-Ara } \\
\text { Glc } & \\
& \text { 2-Rha }\end{array}$ & Et \\
\hline 4 & Glc $\begin{array}{l}\text { 3-Ara } \\
\\
\end{array}$ & Me \\
\hline 5 & $\begin{array}{ll} & \text { 3-Ara } \\
\text { Glc } & \\
& \text { 2-Rha }-A r a\end{array}$ & Et \\
\hline 6 & Glc $\begin{array}{l}\text { 3-Ara } \\
2-\mathrm{Rha}_{3}-\mathrm{Xyl}\end{array}$ & Et \\
\hline 7 & $\begin{array}{ll}\text { Glc } & \text { 3-Ara } \\
& \text { 2-Rha }-X y l\end{array}$ & Me \\
\hline 8 & $\begin{array}{ll} & \text { 3-Rha } \\
\text { Glc } & \\
& \text { 2-Rha }- \text { Ara }\end{array}$ & Et \\
\hline 9 & Glc $\begin{array}{l}\text { 3-Rha } \\
\text { 2-Rha }- \text { Ara }\end{array}$ & Me \\
\hline 10 & Glc $_{6}$-Rha & $\mathbf{E t}$ \\
\hline
\end{tabular}

Abbreviations: Glc: $\beta$-D-Glucopyranosyl

Rha: $\alpha$-L-hamnopyranosyl

Ara: $\alpha$-L-rabinopyranosyl $X y l: \beta-D$ Xylopyranosyl 
Figure 2: Structure of sapimusaponins A-B and O-P

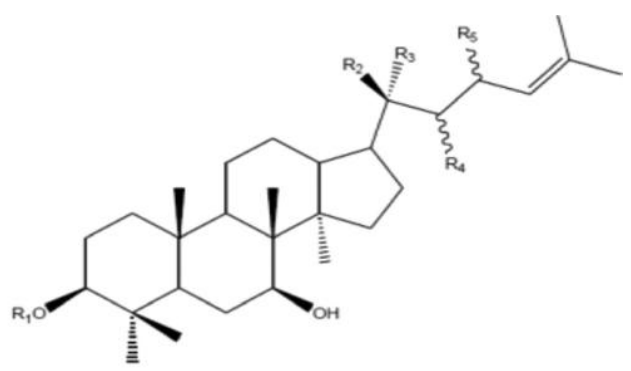

\begin{tabular}{llllll}
\hline Fig & R1 & R2 & R3 & R4 & R5 \\
\hline 1 & Glc - Rha & H & OH & OH & H \\
12 & Glc $_{2}$-Rha & H & OH & OH & OH \\
13 & Glc $_{2}$-Rha & OH & CH & H & H \\
14 & Glc $_{2}$-Rha & CH & OH & H & H \\
\hline
\end{tabular}

Figure 3: Structure of sapimusaponins C-E

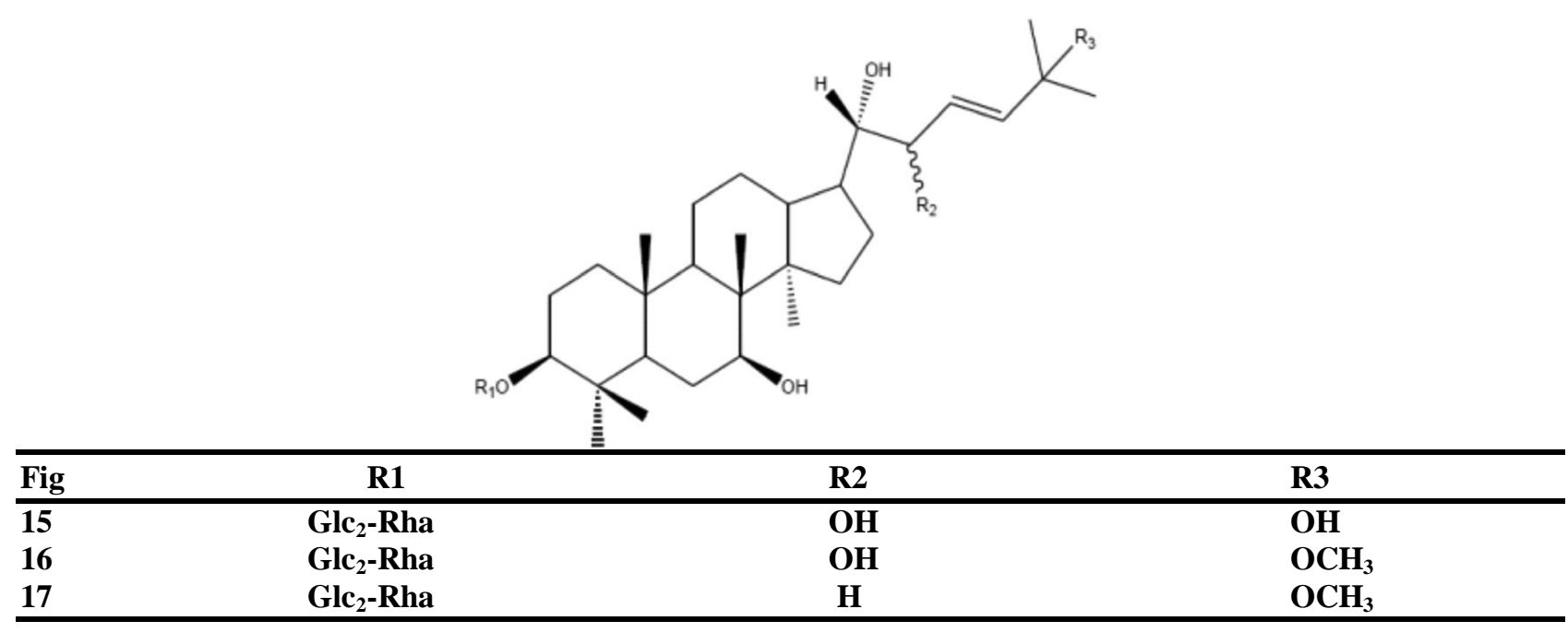

Figure 4: Structure of sapimusaponins F-J and Q-R

\begin{tabular}{|c|c|c|}
\hline Fig & R1 & R2 \\
\hline 18 & Glc $_{6}$-Rha & $\overline{\beta-O C H}{ }_{3}$ \\
\hline 19 & Glc $_{6}-$ Rha & $\alpha-\mathrm{OCH}_{3}$ \\
\hline 20 & $\begin{array}{r}\text { Glc }_{2} \text {-Rha } \\
\text { 2-Rha }\end{array}$ & $\alpha-\mathrm{OCH}_{3}$ \\
\hline 21 & $\begin{array}{l}\text { Glc } \\
\begin{array}{r}\text { 6-Rha } \\
\text { 2-Rha }\end{array}\end{array}$ & $\beta-\mathrm{OCH}_{3}$ \\
\hline 22 & $\begin{array}{l}\text { Glc } \\
\text { 6-Rha }\end{array}$ & $\alpha-\mathrm{OCH}_{3}$ \\
\hline 23 & $\begin{array}{c}\text { Glc }_{2} \text {-Glc } \\
\text { 2-Glc }\end{array}$ & $\alpha-\mathrm{OCH}_{3}$ \\
\hline 24 & $\begin{array}{l}\text { Glc } \\
\text { 6-Rha }\end{array}$ & $\alpha-\mathrm{OCH}_{3}$ \\
\hline
\end{tabular}


Figure 5: Structure of sapimusaponins K-N, sapindosides A-E, mukorozi saponin E1, G, Y1, Y2 and X

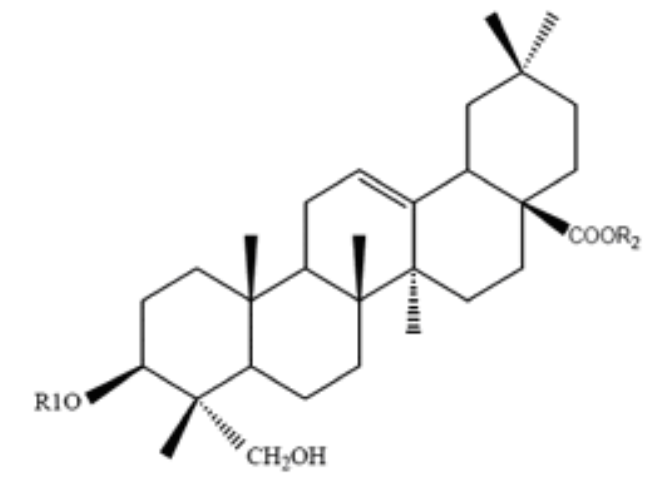

\begin{tabular}{|c|c|c|}
\hline Fig & $\mathbf{R} 1$ & $\mathbf{R 2}$ \\
\hline 25 & Ara $_{2}-$ Rha $_{3}-\mathrm{Ara}_{3}-\mathrm{OAC}$ & $\mathbf{H}$ \\
\hline 26 & $\begin{array}{r}\mathrm{Ara}_{2}-\mathrm{Rha}_{3}-\mathrm{Rha}_{4}-\mathrm{OAC} \\
2-\mathrm{O}\end{array}$ & $\mathbf{H}$ \\
\hline 27 & $\begin{array}{cl}\text { Ara }_{2}-\mathrm{Rha}_{3}-\mathrm{Xyl} & \\
& 3-\mathrm{O} \\
& 2-\mathrm{O}\end{array}$ & $\mathbf{H}$ \\
\hline 28 & $\begin{array}{cc}\operatorname{Ara}_{2}-\mathrm{Rha}_{3}-\mathrm{Xyl} & \\
& \begin{array}{l}4-\mathrm{O} \\
3-O\end{array}\end{array}$ & $\mathbf{H}$ \\
\hline 29 & $\operatorname{Ara}_{2}-R_{h} a_{3}-X y$ & $\mathbf{H}$ \\
\hline 30 & $\mathrm{Ara}_{2}-\mathrm{Rha}_{3}-\mathrm{Xyl}_{4}-\mathrm{OAC}$ & $\mathbf{H}$ \\
\hline 31 & $\operatorname{Ara}_{2}-R_{h} a_{3}-X y l$ & Glc $_{2}$-Glc \\
\hline 32 & Ara $_{2}-\mathrm{Rha}_{3}-\mathrm{Xyl}$ & Glc $_{2}$-Glc \\
\hline 33 & Ara $_{2}$-Rha & Glc $_{2}$-Glc \\
\hline 34 & Ara $_{2}$-Rha & $\mathbf{H}$ \\
\hline 35 & $\operatorname{Ara}_{2}-\mathbf{R h a}_{3}-\mathrm{Xyl}$ & $\mathbf{H}$ \\
\hline 36 & $\operatorname{Ara}_{2}-\mathrm{Rha}_{3}-\mathrm{Xyl}_{4}-\mathrm{Glc}$ & $\mathbf{H}$ \\
\hline 37 & $\operatorname{Ara}_{2}-\mathrm{Rha}_{3}-\mathrm{Xyl}_{4}-\mathrm{Glc}$ & $\mathbf{H}$ \\
\hline 38 & $\operatorname{Ara}_{2}-R_{h a}-X y l$ & $\begin{array}{c}\text { 6-Rha } \\
\operatorname{Ara}_{2}-\mathrm{Rha}_{3}-\mathrm{Xyl}_{4}-\mathrm{Glc} \\
2-\text { Glc }\end{array}$ \\
\hline
\end{tabular}

\section{BIOLOGICAL ACTIVITIES}

The traditional medicinal applications of Sapindus species have inspired many biological investigations. Several extracts of Sapindus species and isolated compounds have been evaluated for their anti-microbial, insecticidal, spermicidal, anti-cancer, hepatoprotective, anxiolytic, tyrosinase inhibition and free radical scavenging property, anti-inflammatory, anti-platelet aggregation, anti-hyperlipidemic, anti-migraine, CNS activity, anti-diabetic, anti-ulcerogenic, analgesic, antiasthmatic, anti-trichomonas, molluscicidal and piscicidal activities $4,7,12,16-18$.

\section{Anti-microbial activity}

Several studies have demonstrated that Sapindus species possess anti-microbial effects in-vivo and in-vitro. Leaf extracts of Sapindus species were reported anti-microbial activity against bacteria. Both methanolic and aqueous extract showed varying degree of inhibitory potential against Escherichia coli and Psuedomonas aeruginosa. Methanolic extract of leaves also showed the antifungal activity against the tested fungus Aspergillus niger. Sapindua showed strong antibacterial activity against $M$. flavus, S. epidermidis and P. morganii ${ }^{15}$. Another investigation was also reported on the leaf extract against six bacterial strains, Pseudomonas testosteroni, Staphylococcus epidermis, Klebsiella pneumonia, Bacillus subtilis and Proteus morganii and showed most potent antibacterial activity ${ }^{38}$. The crude extract of Sapindus mukorossi exhibits a strong growth inhibition against the pathogenic yeast Candida albicans, which causes cutaneous candidiasis. Extracts from the dried pericarp of Sapindus fruits were investigated for their antifungal activity against clinical isolates of yeasts Candida albicans and Candida non-albicans from vaginal secretions of women with Vulvovaginal Candidiasis. Four clinical isolates of C. albicans, a single clinical isolate of each of the species $C$. parapsilosis, $C$. glabrata, $C$. tropicalis, and the strain of $C$. albicans ATCC 90028 were used. The hydroalcoholic extract was bioactivity-directed against a clinical isolate of $C$. parapsilosis, and showed strong activity. The $\mathrm{n}-\mathrm{BuOH}$ extract and one fraction showed strong activity against 
all isolates tested ${ }^{39}$. The saponin fraction inhibited the dermatophytic fungi Trichophyton rubrum, Trichophyton mentagrophytes, Sabouraudites canis and Epidermophyton floccosum ${ }^{11}$. Ibrahim et al. evaluated that ethanolic and chloroform extracts of Sapindus mukorossi inhibited the growth of Helicobacter pylori (both sensitive and resistant), at very low concentrations, when given orally for seven days to male wister rats. In the in-vitro study, the isolates show a considerable zone of inhibition at very low concentration $(10 \mu \mathrm{g} / \mathrm{mL})$ and in the in-vivo study the Helicobacter pylori infection was cleared with minimal dose extracts of $2.5 \mathrm{mg} / \mathrm{mL}^{40}$. Lemos and co-workers reported the anti-microbial activity of saponin from the ethyl-acetate fraction of fruits of $S$. saponaria ${ }^{18}$.

Table 3: Biological effects of genus Sapindus

\begin{tabular}{|c|c|c|c|}
\hline Biological Effect & Details & Extracts / compounds & Ref \\
\hline $\begin{array}{l}\text { Anti-microbial } \\
\text { activity }\end{array}$ & $\begin{array}{l}\text { Antibacterial potential against E. coli, P. } \\
\text { aeruginosa, M. flavus, P. morganii, P. } \\
\text { testosterone, K. pneumonia, B. subtilis } \\
\text { Anti-fungal effect against A. niger } \\
\text { Growth inhibition against the pathogenic yeast } \\
\text { C. albicans and C. non-albicans } \\
\text { Inhibit growth of H. Pylori M }\end{array}$ & $\begin{array}{l}\text { Methanolic and aqueous leaf } \\
\text { extract of } S . \text { mukorossi } \\
\text { Methanolic leaf extract of } S \text {. } \\
\text { mukorossi } \\
\text { Alcoholic dried pericarp extract } \\
\text { of } S \text {. mukorossi } \\
\text { Ethanolic and chloroform extract } \\
\text { of } S . \text { mukorossi }\end{array}$ & $\begin{array}{l}15,38 \\
15,38\end{array}$ \\
\hline $\begin{array}{l}\text { Insecticidal } \\
\text { activity }\end{array}$ & $\begin{array}{l}\text { Significant mortality on insects Sitophilus } \\
\text { oryzae and Pediculus humanus }\end{array}$ & Ethanolic extract of S. mukorossi & 43 \\
\hline $\begin{array}{l}\text { Spermicidal } \\
\text { activity }\end{array}$ & Pregnancy failed to occur & $\begin{array}{llll}\text { Fruit pericarp extract of } S . \\
\text { mukorossi }\end{array}$ & $44,45,46$ \\
\hline $\begin{array}{l}\text { Anti-cancer } \\
\text { activity }\end{array}$ & $\begin{array}{l}\text { Cytotoxic effect on human tumor cell lines } \\
\text { (Hepa59T/VGH, NCL, HeLa and Med) } \\
\text { Inhibit proliferation of human breast Cancer } \\
\text { cell lines (SKBR3 and MDA- MB435) }\end{array}$ & $\begin{array}{l}\text { Saponins from galls extract of } S \text {. } \\
\text { mukorossi } \\
\text { Fruit extract of } S . \text { trifoliatus }\end{array}$ & $\begin{array}{l}47,48,49 \\
13\end{array}$ \\
\hline $\begin{array}{l}\text { Hepatoprotective } \\
\text { activity }\end{array}$ & $\begin{array}{l}\text { Protective capacity on primary hepatocytes } \\
\text { culture and rat model of } \mathrm{CCl}_{4} \text { mediated liver } \\
\text { injury }\end{array}$ & $\begin{array}{l}\text { Fruit pericarp extract of } S . \\
\text { mukorossi }\end{array}$ & 50 \\
\hline $\begin{array}{l}\text { Anxiolytic } \\
\text { Activity }\end{array}$ & Significant anxiolytic activity & $\begin{array}{l}\text { Methanolic extract of } S . \\
\text { mukorossi }\end{array}$ & 51 \\
\hline $\begin{array}{l}\text { Tyrosinase } \\
\text { inhibition and free } \\
\text { radical scavenging } \\
\text { activity }\end{array}$ & $\begin{array}{l}\text { Inhibit proliferation of human melanoma and } \\
\text { lung cell lines }\end{array}$ & $\begin{array}{l}\text { Methanolic extract of seeds of } S \text {. } \\
\text { mukorossi }\end{array}$ & 52 \\
\hline $\begin{array}{l}\text { Anti- } \\
\text { inflammatory } \\
\text { activity }\end{array}$ & $\begin{array}{l}\text { Inhibitory effect on granuloma and exudates } \\
\text { formation } \\
\text { Inhibit increase in vascular Permeability } \\
\text { Reduce number of writhing induced- By acetic } \\
\text { acid } \\
\text { Inhibit the hind paw edema-associated with } \\
\text { adjuvant arthritis } \\
\text { Inhibit effect on leucocyte migration } \\
\text { Inhibitory activity against Inflammatory agents } \\
\text { 5-lipoxygenase, cyclooxygenase, Leukotriene } \mathrm{B}_{4} \\
\text { and nitric oxide- Synthase }\end{array}$ & $\begin{array}{l}\text { Ethanolic extract of Seeds of } S \text {. } \\
\text { trifoliatus } \\
\text { Aqueous extract of fruit pericarp } \\
\text { of } S . \text { trifoliatus }\end{array}$ & $\begin{array}{l}53 \\
53 \\
53\end{array}$ \\
\hline
\end{tabular}




\begin{tabular}{|c|c|c|c|}
\hline $\begin{array}{l}\text { Anti-platelet } \\
\text { Aggregation } \\
\text { activity }\end{array}$ & $\begin{array}{l}\text { More potent anti-platelet aggregation activity } \\
\text { that aspirin }\end{array}$ & $\begin{array}{l}\text { Sapinmusaponins Q\&R and F-J } \\
\text { isolated from ethanolic extract of } \\
\text { galls of } S \text {. mukorossi }\end{array}$ & 34 \\
\hline $\begin{array}{l}\text { Anti- } \\
\text { hyperlipidemic } \\
\text { activity }\end{array}$ & Lower plasma glycerides and cholesterol level & Methanolic fruit extract & 56 \\
\hline $\begin{array}{l}\text { Anti-migraine } \\
\text { activity }\end{array}$ & Anti-migraine hyperalgesic potential & $\begin{array}{l}\text { Aqueous extract of pericarp of } S \text {. } \\
\text { trifoliatus }\end{array}$ & 57 \\
\hline CNS activity & $\begin{array}{l}\text { Reduction in spontaneous activity } \\
\text { Reduction in exploratory behavioral pattern } \\
\text { Muscles relaxant activity } \\
\text { Inhibition of cocaine induced Hyperactivity } \\
\text { Potentiate phenobarbitone sodium- induced } \\
\text { sleeping time } \\
\text { CNS depressant action }\end{array}$ & 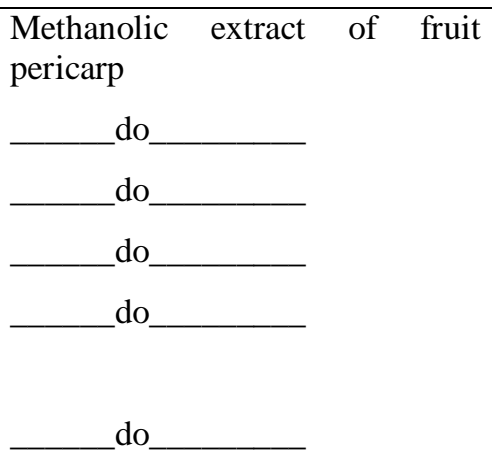 & $\begin{array}{l}58 \\
58 \\
58 \\
58\end{array}$ \\
\hline $\begin{array}{l}\text { Anti-diabetic } \\
\text { activity }\end{array}$ & Significant hypoglycemic effect & Alcoholic extract of leaves & 60 \\
\hline $\begin{array}{l}\text { Anti-ulcerogenic } \\
\text { activity }\end{array}$ & $\begin{array}{l}\text { Reduce gastric secretion } \\
\text { Reduction in pylorus ligation induced ulcers }\end{array}$ & $\begin{array}{l}\text { Hydro-alcoholic extract of leaves } \\
\text { and fruits of S. saponaria } \\
\text { Methanolic and aqueous extract } \\
\text { of leaves of } S \text {. trifoliatus }\end{array}$ & 12 \\
\hline Analgesic activity & Reduce diabetic neuropathic pain & Ethanolic extract of $S$. trifoliatus & 62 \\
\hline $\begin{array}{l}\text { Anti-trichomonas } \\
\text { activity }\end{array}$ & $\begin{array}{l}\text { Inhibit ability of parasites to adhere to HeLa } \\
\text { cells } \\
\text { Decrease proteolytic activity of the parasites } \\
\text { cystein proteinases }\end{array}$ & Saponin mixture & 63,44 \\
\hline $\begin{array}{l}\text { Molluscicidal } \\
\text { activity }\end{array}$ & $\begin{array}{l}\text { Against golden apple snail, } \\
\text { canaliculata and Lymnaea acuminate }\end{array}$ & $\begin{array}{l}\text { extract of fruit pericarp of } S . \\
\text { mukorossi }\end{array}$ & 65,66 \\
\hline Piscicidal activity & High potential for fish eradication & $\begin{array}{l}\text { extract of fruit pericarp of } S . \\
\text { mukorossi }\end{array}$ & 67 \\
\hline $\begin{array}{l}\text { Anti-asthmatic } \\
\text { activity }\end{array}$ & $\begin{array}{l}\text { Inhibit acetylcholine } \\
\text { bronchoconstriction }\end{array}$ & $\begin{array}{l}\text { Aqueous extract of fruit of } S \text {. } \\
\text { mukorossi }\end{array}$ & 68 \\
\hline
\end{tabular}

\section{Insecticidal activity}

Saponins possess insecticidal activity, causing mortality and/or growth inhibition in the insects tested the cotton leafworm Spodoptera littoralis caterpillars and the pea aphid Acyrthosiphon pisum. In the experiments with Acyrthosiphon pisum, $0.1 \%$ saponin killed all aphids, whereas with Spodoptera some caterpillars were still able to develop into apparently normal adults on food containing $7 \%$ saponins $^{41}$. Saponins can be employed as novel natural tactics in integrated pest management (IPM) to control pest insects, which fit in modern agriculture and horticulture ${ }^{42}$. Ethanolic extract of Sapindus mukorossi was investigated for repellency and insecticidal activity against Sitophilus oryzae and Pediculus humanus. Average mortality percentage indicated that the extracts caused significant mortality and repellency on the target insects and bioassays indicated that toxic and repellent effect was proportional to the concentration ${ }^{43}$.

\section{Spermicidal activity}

Saponins from Sapindus mukorossi are known to be spermicidal ${ }^{44}$. Morphological changes in human ejaculated spermatozoa after exposure to this saponin were evaluated under scanning electron microscopy. The minimum effective concentration $(0.05 \%$ in spot test $)$ did not affect the surface topography after exposure for one minute. However, incubation of spermatozoa for 10 minutes resulted in extensive vesiculation and a disruption of the plasma membrane in the head region. Higher concentrations $(0.1 \%, 1.25 \%, 2.5 \%$ and $5.0 \%)$ caused more or less similar changes which included vesiculation, vacuolation, disruption or erosion of membranes in the head region. These findings suggest that the morphological changes observed are due to 
alterations in the glycoproteins associated with the lipid bilayer of the plasma membrane of spermatozoa ${ }^{8}$. This spermicidal property has been used in contraceptive cream ${ }^{45}$. A contraceptive cream developed by the CDRI (Lucknow) is going to hit the Indian markets. It is advocated to be totally safe and easy to use. It is intended for post-coital use ${ }^{46}$. Raghuvanshi et al. developed a spermicide compound, called Praneem polyherbal, featuring anti-microbial traits, from the pericarp of fruit of S. mukorossi, leaves of Azadirachta indica, and oil of Mentha citrata. The association of these three plants produced a highly powerful spermicide, which was tested on rabbits and on human sperm through in-vitro and in-vivo studies. When this spermicide was applied to the vagina of female rabbits, pregnancy failed to occur ${ }^{18}$.

\section{Anti-cancer activity}

Due to the great variability in saponin structure, saponins always display anti-tumorigenic effect through varieties of anti-tumor pathways ${ }^{47}$. Kuo and co-workers tested the cytotoxic effect of saponins isolated from the galls of $S$. mukorossi. The preliminary bioassay data revealed that saponins showed moderate cytotoxic activity $\left(\mathrm{ED}_{50} \sim 9\right.$ $18 \mu \mathrm{g} / \mathrm{mL}$ ) against human tumor cell lines (Hepa59T/VGH, NCL, HeLa and Med) ${ }^{48}$. Strychnopentamine was the reference compound used in the study. All saponins were reported to be at least five times less active than the reference compound ${ }^{49}$. Pradhan and co-workers reported that fruit extract of $S$. trifoliatus inhibit the proliferation of human breast cancer cell lines SKBR3 and MDA-MB435 ${ }^{13}$.

\section{Hepatoprotective activity}

Ibrahim and co-workers reported that the extracts of fruit pericarp of Sapindus mukorossi $(2.5 \mathrm{mg} / \mathrm{L})$ and rhizomes of Rheum emodi $(3.0 \mathrm{mg} / \mathrm{L})$ have a protective capacity both in-vitro on primary hepatocytes cultures and in-vivo in a rat model of carbon tetrachloride $\left(\mathrm{CCl}_{4}\right)$ mediated liver injury as judged from serum marker enzyme activities ${ }^{50}$. Thus, it was concluded that the extracts of Sapindus mukorossi and Rheum emodi do have a protective capacity both in-vitro on primary hepatocytes cultures and in in-vivo in a rat model of $\mathrm{CCl}_{4}$ mediated liver injury.

\section{Anxiolytic activity}

Methanolic extracts of $S$. mukorossi (200 and $40 \mathrm{mg} / \mathrm{L}$ ) show significant anxiolytic activity as compared to standard anxiolytics Diazepam $(2 \mathrm{mg} / \mathrm{Kg})$ and Fluoxetine $(10 \mathrm{mg} / \mathrm{Kg})^{51}$.

\section{Tyrosinase inhibition and free radical scavenging activity}

Chen et al. first evaluated that the extracts of $S$. mukorossi seeds using methanol $(\mathrm{MeOH})$, ethyl acetate (EA) or hexane as solvents show tyrosinase inhibition and free radical scavenging properties. S. mukorossi extracts showed strong specific inhibition activities on the proliferation of human melanoma and lung cell lines. The data exhibited the high potential of applying $S$. mukorossi extracts in medical cosmetology, food supplementation, antibiotics and chemotherapy ${ }^{52}$.
Takagi and co-workers investigated the antiinflammatory activities of hederagenin and crude saponin isolated from $S$. mukorossi, using carrageenininduced edema, granuloma pouch and adjuvant arthritis in rats. The effects of these agents on vascular permeability and acetic-acid-induced writhing in mice were also examined. Anti-inflammatory activity on carrageenin edema was observed after intraperitoneal and oral administration of crude saponin, whereas hederagenin and the other agents showed activity only when administered intraperitoneally. They observed that crude saponin showed a significant inhibitory effect on granuloma and exudate formations in rats, inhibited the increase in vascular permeability and the number of writhings induced by acetic acid in mice, and significantly inhibited the development of hind-paw edema associated with adjuvant arthritis in rats after oral administration. They concluded that crude saponin shows some degree of anti-inflammatory activity ${ }^{53}$. Arul and co-workers reported the anti-inflammatory action of an ethanol extract of $S$. trifoliatus seeds by paw-edema induction and pleurisy methods caused by carrageenin and granuloma formation. The extract produced decreases in paw edema and in pleural sweating volume, and had an inhibitory effect on leucocyte migration. A decrease in granuloma weight was also reported ${ }^{54}$. Arulmozhi and co-workers investigated the effect of the aqueous lyophilized extract of the pericarp of $S$. trifoliatus fruits through in-vivo and in-vitro experimental models of inflammation. The in-vitro evaluation showed the extract inhibitory activity against the inflammatory agents 5-lipoxygenase, cyclooxygenase, leukotriene B4 and nitricoxide synthase. The extract significantly inhibited inflammation of paw edema caused by carrageenin, histamine, serotonin and zymosan in rats and mice. Moreover, topical application significantly inhibited ear edema caused by inflammatory agents such as 13-acetate-O-tetradecanoilphorbol (TPA), capsaicin or arachidonic acid. They concluded that the extract has an anti-inflammatory activity, probably mediated by the 5-lipoxygenase and cyclo-oxygenase pathways ${ }^{55}$.

\section{Anti-platelet aggregation activity}

Biological evaluation of ethanolic extract of the galls of $S$. mukorossi showed that two saponins isolated sapinmusaponins $\mathrm{Q}$ and $\mathrm{R}$ demonstrated more potent anti-platelet aggregation activity than aspirin ${ }^{34}$. Sapinmusaponins F-J isolated from the galls of $S$. mukorossi showed anti-platelet-aggregation effects, but no obvious cytotoxic activity for platelets as assayed by lactate dehydrogenase (LDH) leakage was reported ${ }^{31}$.

\section{Anti-hyperlipidemic activity}

The saponins from Sapindus species fruit extract found to have significant anti-hyperlipidemic activity. Methanolic extract at a dose of 100 and $200 \mathrm{mg} / \mathrm{kg}$ significantly lowered both plasma glycerides and cholesterol levels. The cholesterol lowering activity of the fruit extract result from the breakdown of LDL cholesterol by enhancing enzymatic action ${ }^{56}$.

\section{Anti-migraine activity}


Arulmozhi and co-workers studied the effect of the aqueous pericarp extract of fruits of $S$. trifoliatus in an in-vivo migraine hyperalgesic model. The results showed that antagonism to dopamine D2 might underlie the mechanism involved in the anti-hyperalgesic activity of the plant extract ${ }^{57}$.

\section{CNS activity}

Methanolic extract of fruit of Sapindus species found to produce CNS depressant activity. The methanolic extract of Pericarps at 100 and $200 \mathrm{mg} / \mathrm{kg}$ caused a significant reduction in the spontaneous activity, exploratory behavioral pattern, muscle relaxant activity, inhibition of cocaine induced hyperactivity and also extensively potentiated phenobarbitone sodium-induced sleeping time. This extract produced a considerable increase in the hypnotic effect induced by the phenobarbitone in a dose dependent manner, suggesting a profile sedative activity 58. Various scientific research reports showed that presence of triterpenoids in the methanolic extract may be responsible for the CNS depressant activity ${ }^{59}$.

\section{Anti-diabetic activity}

Jeyabalan and co-workers studied the anti-hyperglycemic effect of alcoholic extract of leaves of Sapindus species in the glucose overloaded hyperglycemic rats. The extract at different doses exhibited a significant hypoglycemic activity in dose dependent manner. The study also revealed that level of total hemoglobin, glycosylated hemoglobin, serum creatinine, serum urea and lipid profiles measured showed the anti-diabetic activity ${ }^{60}$.

\section{Anti-ulcerogenic activity}

Albiero and co-workers investigated the inhibitory effect of a hydro-alcoholic extract of leaves and fruits of $S$. saponaria on stress-induced gastric lesions. Their results showed that both extracts caused a decrease in gastric secretion. However, the saponin- and tannin-rich fruit extract was more effective in anti-ulcerogenic activity ${ }^{61}$. In another study, Kishore and co-workers reported that methanolic and aqueous extracts of leaves of $S$. trifoliatus showed significant reduction in the pylorus ligation induced ulcers in rats ${ }^{12}$.

\section{Analgesic activity}

Sahoo and co-workers investigated the ethanolic extract of Sapindus trifoliatus in in-vivo model of diabetic neuropathic pain. They concluded that extract showed significant effectiveness in diabetic neuropathic pain and protection produced by stimulation of adenosine A1receptors 62 .

\section{Anti-trichomonas activity}

Tiwari and co-workers demonstrated that the Sapindus saponin mixture shows anti-Trichomonas activity at a 10 -fold lower concentration $(0.005 \%)$ than its minimal effective spermicidal concentration against human spermatozoa $(0.05 \%){ }^{63}$. Saponin concentration dependently inhibited the ability of parasites to adhere to HeLa cells and decreased the proteolytic activity of the parasite's cysteine proteinases. This was associated with the decreased expression of adhesin AP65 and membrane-expressed cysteine proteinase TvCP2 genes.
Saponins produced no adverse effect on host cells in mitochondrial reduction potential measurement assay. Saponins also reversed the inhibitory mechanisms exerted by Trichomonas for evading host immunity. Early response of saponins to disrupt actin cytoskeleton in comparison with their effect on the nucleus suggests a membrane-mediated mode of action rather than via induction of apoptosis 44,63 .

\section{Molluscicidal activity}

Extracts of Sapindus mukorossi showed molluscicidal effect against the golden apple snail and Pomacea canaliculata Lamarck. (Ampullariidae) with $\mathrm{LC}_{50}$ values of 85,22 and $17 \mathrm{ppm}$ at 24, 48 and $72 \mathrm{~h}$ exposure period, respectively ${ }^{64}$. Upadhyay and Singh, reported that $S$. mukorossi fruit pericarp is a potential source of botanical molluscicides against Lymnaea acuminate ${ }^{65}$. These snails are the intermediate host of liver fluke Fasciola gigantica, which causes $94 \%$ fascioliasis in the buffalo population of northern India ${ }^{66}$. Ribeiro and co-workers reported molluscicidal activity of alcoholic extract of Sapindus saponaria ${ }^{18}$.

\section{Piscicidal activity}

Effects of Sapindus mukorossi have been studied on fish. Pericarp of $S$. mukorossi is the most toxic parts yielding $100 \%$ mortality rate within 12 hours and mean survival time was found to be 1.18 hours. $\mathrm{LD}_{10}, \mathrm{LD}_{50}, \mathrm{LD}_{100}$ ranging between $3.5 \mathrm{ppm}$ and $10 \mathrm{ppm}$ at $48 \mathrm{hrs}$ and possess high potential for fish eradication. S. mukorossi fruit pericarp can be used as a selective eradicant for horny fish like Heteropneustes fossils and Channa punctuate $^{67}$.

\section{Anti-asthmatic activity}

Parganiha and co-workers evaluated the in-vitro antiasthmatic activity of Sapindus mukorossi on acetylcholine induced contraction of goat tracheal chain preparation. They reported that the aqueous extract of $S$. mukorossi fruits at the doses of $380 \mathrm{mcg} / \mathrm{ml}$ and 640 $\mathrm{mcg} / \mathrm{ml}$ significantly inhibited acetylcholine induced bronchoconstriction of isolated goat trachea and concluded that the aqueous extract of fruit of $S$. mukorossi has significant anti-asthmatic potential ${ }^{68}$.

\section{CONCLUSIONS}

In this review, we documented the existing traditional uses of the species of the genus Sapindus and summarized recent research in to the phytochemistry and biological properties of the genus. Previous studies have documented that Sapindus species have traditionally been used to treat ulcers, external wounds, inflammation, excessive salivation, pimples, epilepsy, chlorosis, migraines, eczema, psoriasis, dental caries, arthritis, common colds, constipation, nausea, joint pain, gout and rheumatism. Some of these traditional uses have been validated by phytochemical and modern pharmacological studies. The extracts and single compounds derived from the genus have been found to possess various biological activities, especially in the areas of anti-microbial, insecticidal, spermicidal, anti-cancer, hepatoprotective, anxiolytic, tyrosinase inhibition and free radical scavenging property, anti-inflammatory, anti-platelet aggregation, anti-hyperlipidemic, anti-migraine, CNS 
activity, anti-diabetic, anti-ulcerogenic, analgesic, antiasthmatic, anti-trichomonas, molluscicidal and piscicidal activities. Triterpenoidal saponins of oleanane, dammarane and tirucullane types are regarded as the active group that is most likely to be responsible for the observed biological properties.

Although increased interest has prompted more studies on the phytochemistry and biological activities of the genus Sapindus, there are still many areas where our current knowledge could be improved. (i) Several traditional uses of the genus have been validated in recent biological studies; however, some of these biological activities were only tested in-vitro. Thus, the effectiveness of these compounds in-vivo needs to be further investigated. (ii) The genus Sapindus is a rich source of triterpenoidal saponins of oleanane, dammarane and tirucullane, many with the same skeletal structure. Therefore, it would be interesting to investigate the structure-activity relationships of these saponins. We would expect to find high efficiency compounds from these saponins. (iii) It is evident from the available

\section{REFERENCES}

1. Venkatesh V, Sharma JD, Kamal R, A comparative study of effect of alcoholic extracts of Sapindus emarginatus, Terminalia belerica, Cuminum cyminum and Allium cepa on reproductive organs of male albino rats, Asian J Exp Sci, 2002, 16(1-2), 51-63

2. Mahmood A, Asif IM, Alam MS, Islam MS, Eti SA, Hossain F, Extraction and characterization of oils from Sapindus trifoliatus Linn seeds of different origin of Bangladesh, Merit Res J Environ Sci Toxicol, 2013, 1(5), 99-104.

3. Vora RS, Seed germination characteristics of selected native plants of the lower Rio Grande Valley, Texas, J Range Manage, 1989, 42(1), 36-40.

4. Upadhyay A, Singh DK, Pharmacological effects of Sapindus mukorossi, Rev Inst Med Trop Sao Paulo, 2012, 54(5), 273280.

5. Meena VN, Rajakohila M, Syndia LAM, Prasad PN, Ariharan VN, Multifacetious uses of Soapnut Tree - A mini review, RJPBCS, 2012, 3(1), 420-424.

6. Albiero ALM, Bacchi EM, Mourao KSM, Caracterizacao anatomica das folhas, frutos esementes de Sapindus saponaria L. (Sapindaceae), Acta Scientiarum, 2001, 23, 549-560.

7. Sharma A, Sati SC, Sati OP, Sati D, Kothiyal SK, Chemical constituents and bioactivities of genus Sapindus, Int J Res Ayurveda Pharm, 2011, 2, 403-409.

8. Dhar JP, Bajpai VK, Setty BS, Kamboj VP, Morphological changes in human spermatozoa as examined under scanning electron microscope after in vitro exposure to saponins isolated from Sapindus mukorossi, Contraception, 1989, 39, 563-568.

9. Kasai R, Fujino H, Kuzuki T, Wong WH, Goto C, Yata N, Acyclic sesquiterpene oligoglycosides from pericarps of Sapindus mukorossi, Phytochemistry, 1986, 25, 871-876.

10. Singh N, Kaur A, Yadav K, A reliable in-vitro protocol for rapid mass propagation of Sapindus mukorossi Gaertn, Nat Sci, 2010, 8, 41-47.

11. Tanaka O, Tamura Y, Masuda H, Mizutani K. Saponins used in food and agriculture. New York: Plenum Press; 1996. P. 1-11.

12. Kishore DV, Pinto J, Mini KV, Ronald F, Satyanarayana D, Anti ulcer activity of leaf extract of Sapindus trifoliatus Linn, IJAPS, 2010, 1(1), 104-107.

13. Rao GHJ, Lakshmi P, Sapindus trifoliatus: A Review, IJPT, 2012, 4(3), 2201-2204.

14. Arulmozi DK, Veeranjaneyulu A, Bodhankar SL, Arora SK, Investigations of Sapindus trifoliatus in dopaminergic and serotonergic systems, purative anti migraine mechanisms, Ind J Pharmacol, 2005, 37, 120-125. literature that Sapindus species possess potential for use as a beneficial therapeutic remedy. However, the analysis of previous biological research suggests that a clear assignment of active molecules and mechanisms of action is remain lacking. Taken together, the importance of genus Sapindus has been highlighted based on their wide usage in traditional medicine as well as potential in beneficial therapeutic remedy. Nevertheless, there is clearly a need for further studies focusing on in-vivo with emphasis on molecular mechanisms and eventually clinical trials.

\section{ACKNOWLEDGEMENTS}

This work was supported by the Department of Science and Technology, Government of Rajasthan (India) [F7 (7) ST/SP/2013/3194].

\section{DECLARATION OF INTEREST}

The authors report no conflicts of interest. The authors alone are responsible for the content and writing of this article.

15. Deepa T, Elamanthi R, Kavitha R, Kumar S, Screening for Physical Phytochemical and Antimicrobial Activities of leaf extracts of Sapindus emarginatus Vahl, IJPTR, 2012, 4(1), 392-397.

16. Suhagia BN, Rathod IS, Sindhu S, Sapindus mukorossi (Areetha): An overview, IJPSR, 2011, 2(8), 1905-1913.

17. Arora B, Bhadauria P, Tripathi D, Sharma A, Sapindus emarginatus: Phytochemistry \& various biological activities, IGJPS, 2012, 2(3), 250-257.

18. Pelegrini DD, Tsuzuki JK, Amado CAB, Cortez DAG, Ferreira ICP, Biological activity and isolated compounds in Sapindus saponaria L. and other plants of the genus Sapindus, Lat Am J Pharm, 2008, 27(6), 922-927.

19. Khatamian H, Abuelgasim Z, Auxins aid soapberry cuttings, American Nurseryman, 1986, 164 (4), 65-72.

20. Munson RH, Germination of western soapberry as affected by scarification and stratification, Hort Science, 1984, 19 (5), 712713.

21. Kommalapati RR, Valsaraj KT, Constant W, Roy D, Soil flushing using colloidal gas aphron suspensions generated from a plant-based surfactant, J Hazardous Materials, 1998, 60 (1), 73-87.

22. Krishnaveni A, Thaakur SR, Pharmacognostical and preliminary phytochemical studies of Sapindus trifoliatus Vahl, Ethnobotanical Leaflets, 2008, 12, 820-826.

23. Francis G, Kerem Z, Makkar H, Becker K, The biological action of saponins in animal systems: a review, Br $\mathrm{J}$ Nutr, 2002, 88, 587-605.

24. Saxena D, Pal R, Dwivedi AK, Singh S, Characterization of sapindosides in Sapindus mukorossi saponin (Reetha saponin) and quantitative determination of sapindoside B, J Sci Ind Res, 2004, 63, 181-186.

25. Vincken JP, Heng L, Gruppen H, Saponins, classification and occurrence in the plant kingdom, Phytochemistry, 2007, 68, 275-297.

26. Chirva V, Kintya P, Sosnovskii VA, Krivenchuk PE, Zykova NY, Triterpene glycosides of Sapindus mukorossi. II. The structure of Sapindoside A \& B, Chem Nat Compounds, 1970, 6, 213-215.

27. Chirva V, Kintya PK, Sosnovskii VA, Triterpene glycosides of Sapindus mukorossi. III. The structure of sapindoside C, Chem Nat Compounds, 1970, 6, 380-381

28. Chirva V, Kintya PK, Sosnovskii VA, Zolotarev BM, Triterpene glycosides of Sapindus mukorossi. IV. The structure of sapindoside D, Chem Nat Compounds, 1970, 6, 316-318. 
29. Chirva V, Kintya PK, Sosnovskii VA, Triterpene glycosides of Sapindus mukorossi. V. The structure of sapindoside E, Chem Nat Compounds, 1970, 6, 440-442.

30. Yao HK, Hui CH, Li-Ming YK, Ya-Wen H, Kuo-Hsiung L, Fang-Rong C, New dammarane-type saponins from the galls of Sapindus mukorossi, J Agric Food Chem, 2005, 53, 4722-4727.

31. Huang HC, Tsai WJ, Morris-Natschke SL, Tokuda H, Lee KH, $\mathrm{Wu}$ YC, Sapinmusaponins F-J, bioactive tirucallane-type saponins from the galls of Sapindus mukorossi, J Nat Prod, 2006, 69, 763-767.

32. Huang HC, Wu MD, Tsai WJ, Liao SC, Liaw CC, Hsu LC, Triterpenoid saponins from the fruits and galls of Sapindus mukorossi, Phytochemistry, 2008, 69, 1609-1616.

33. Nakayama K, Fujino H, Kasai R, Mitoma Y, Yata N, Tanaka O, Solubilizing properties of saponins from Sapindus mukorossi Gaertn, Chem Pharm Bull (Tokyo), 1986, 34, 32793283.

34. Huang HC, Tsai WJ, Liaw CC, Wu SH, Wu YC, Kuo YH, Anti-platelet aggregation triterpene saponins from the galls of Sapindus mukorossi, Chem Pharm Bull (Tokyo), 2007, 55(9), 1412-1415.

35. Teng RW, Ni W, Hau Y, Chen CX, Two new tirucallane-type triterpenoid saponins from Sapindus mukorossi, Acta Botanica Sinica, 2003, 45, 369-372.

36. Ni W, Hua Y, Teng RW, Kong YC, Chen CX, New tirucallane-type triterpenoid saponins from Sapindus mukorossi, J Asian Nat Prod Res, 2004, 6, 205-209.

37. Ni W, Hua Y, Liu HY, Teng RW, Kong YC, Hu XY, Tirucallane-type triterpenoid saponins from the roots of Sapindus mukorossi, Chem Pharm Bull (Tokyo), 2006, 54, 1443-1446.

38. Nair R, Kalariya T, Chanda S, Antibacterial activity of some selected Indian medicinal flora, Turk J Biol, 2005, 29, 41-47.

39. Tsuzuki JK, Svidzinski TIE, Shinobu CS, Silva LFA, Rodrigues-Filho E, Cortex DAG, Antifungal activity of the extracts and saponins from Sapindus saponaria, Annals of the Brazilian Academy of Sciences, 2007, 79(4), 577-583.

40. Ibrahim M, Khan AA, Tiwari SK, Habeeb MA, Khaja MN, Habibullah CM, Anti-microbial activity of Sapindus mukorossi and Rheum modi extracts against Helicobacter pylori: in-vitro and in-vivo studies, World J Gastroenterol, 2006, 12, 71367142

41. Geyter ED, Geelen D, Smagghe G, First results on the insecticidal action of saponins, Commun Agric Appl Biol Sci, 2007, 72, 645-648.

42. Geyter ED, Lambert E, Geelen D, Smagghe G, Novel advances with plant saponins as natural insecticides to control pest insects, Pest Tech, 2007, 1, 96-105.

43. Rahman SS, Rahman M, Begum SA, Khan MMR, Bhuiyan $\mathrm{MH}$, Investigation of Sapindus mukorossi extracts for repellency, insecticidal activity and plant growth regulatory effect, J Appl Sci Res, 2007, 3, 95-101.

44. Garg S, Taluja V, Upadhyay M, Talwar GP, Studies on contraceptive efficacy of Praneem polyherbal cream, Contraception, 1993, 48, 591-596.

45. Dwivedi AK, Chaudhry M, Sarin JPS, Standardization of a new spermicidal agent Sapindus saponin and its estimation in its formulation, Indian J Pharm Sci, 1990, 52, 165-167.

46. Maikhuri JP, Dwivedi AK, Dhar JD, Shetty BS, Gupta G, Mechanism of action of some acrylophenones, quinolones and dithiocarbamate as potent, non-detergent spermicidal agents, Contraception, 2003, 67(5), 403-408.

47. Man S, Gao W, Zhang Y, Huang L, Liu C, Chemical study and medical application of saponins as anti-cancer agents, Fitoterapia, 2010, 81, 703-714.

48. Sengupta A, Basu SP, Saha S, Triglyceride composition of Sapindus mukorossi seed oil, Lipids, 1975, 10, 33-40.

49. Takechi M, Tanaka Y, Structure-activity relationships of the saponin $\alpha$-hederin. Phytochemistry, 1990, 29, 451-452.
50. Ibrahim M, Khaja MN, Aara A, Khan AA, Habeeb MA, Devi YP, Hepatoprotective activity of Sapindus mukorossi and Rheum modi extracts: in-vitro and in-vivo studies, World J Gastroenterol, 2008, 14, 2566-2571.

51. Chakraborty A, Amudha P, Geetha M, Surjit Singh N, Evaluation of anxiolytic activity of methanolic extract of Sapindus mukorossi Gaertn in mice, Int J Pharm Bio Scie, 2010, 1, 1-8.

52. Chen CY, Kuo PL, Chen YH, Huang JC, Ho ML, Lin RJ, Tyrosinase inhibition, free radical scavenging, antimicroorganism and anti-cancer proliferation activities of Sapindus mukorossi extracts, J Taiwan Inst Chem Engrs, 2010, 41, 129-135.

53. Takagi K, Park EH, Kato H, Anti-inflammatory activities of hederagenin and crude saponin isolated from Sapindus mukorossi Gaertn, Chem Pharm Bull, 1980, 28, 1183-1188.

54. Arul B, Kothai R, Jacob P, Anti-inflammatory activity of Sapindus trifoliatus Linn, J Herb Pharmacother, 2004, 4(4), 4350

55. Arulmozhi DK, Veeranjaneyulu A, Bodhankar SL, Arora SK, Pharmacological investigations of Sapindus trifoliatus in various in-vitro and in-vivo models of inflammation, Indian $\mathrm{J}$ Pharmacol, 2005, 37(2), 96-102.

56. Jeyabalan S, Palayan M, Antihyperlipidemic activity of Sapindus emarginatus in Triton WR-1339 induced albino rats, Research J Pharm Tech, 2009, 2 (2), 319-323.

57. Arulmozhi DK, Veeranjaneyulu A, Bodhankar SL, Arora SK, Effect of Sapindus trifoliatus on hyperalgesic in-vivo migraine models, Braz J Med Biol Res, 2005, 38, 469-475.

58. Srikanth J, Muralidharan P, CNS activity of the methanolic extracts of Sapindus emarginatus Vahl in experimental models, J Sci Res, 2009, 1(3), 583-593.

59. Chattopadhyay D, Arunachalam G, Mandal AB, Bhadra R, Mandal SC, CNS activity of Mallotus peltatus Muell Arg. leaf extracts. An ethnomedicine of Bay Islands. J Ethnopharmacology, 2003, 85-99.

60. Srikanth J, Muralidharan P, Anti-hyperglycemic and antidiabetic activity of leaves extracts of Sapindus emarginatus Vahl, Asian Biomedicine, 2009, 3(3), 313-318.

61. Albiero ALM, Sertie JAA, Bacchi EM, Antiulcer activity of Sapindus saponaria L. in the rat, J Ethnopharmacol, 2002, 82(1), 41-44.

62. Sahoo PK, Pradhan D, Behra P, Pharmacological interventions of some potential herbal extracts targeted at adenosine receptors in diabetic neuropathic pain, J Pharmacy Research, 2010, 3(8), 1798-1802.

63. Tiwari P, Singh D, Singh MM, Anti-Trichomonas activity of Sapindus saponins, a candidate for development as microbicidal contraceptive, J Antimicrob Chemother, 2008, 62, 526-534.

64. Huang HC, Liao SC, Chang FR, Kuo YH, Wu YC, Molluscicidal saponins from Sapindus mukorossi, inhibitory agents of Golden Apple snails Pomacea canaliculata, J Agric Food Chem, 2003, 51, 4916-4919.

65. Upadhyay A, Singh DK, Molluscicidal activity of Sapindus mukorossi and Terminalia chebula against the freshwater snail Lymnaea acuminata. Chemosphere, 2011, 83, 468-474.

66. Singh DK, Agarwal RA, In-vivo and in-vitro studies on synergism with anticholinesterase pesticides in the snail Lymnaea acuminate, Arch Environ Contain Toxicol, 1983, 12, 483-487.

67. Virdi GS, The piscicidal properties of Acorus calamus, Sapindus mukorossi and Xeromphis spinosa on 7 species of fishes of North India, Indian J Phys Nat Sci, 1982, 2, 28-35.

68. Parganiha R, Mishra J, Deshmukh VS, Muley BP, Sawarkar HA, Kashyap P, In- vitro anti- asthmatic activity of fruit extract of Sapindus mukorossi, International Journal Herbal Drug Research, 2011, 1(1), 11-14. 